Thus although correlations between geomagnetic changes and seismic activity have been reported quite frequently since 1799 (von Humboldt), few, if any, can be considered genuine. The problems of sensitivity and vibration have now been largely overcome in modern equipment; and noise from distant sources such as the ionosphere can be filtered out by coupling local magnetometers with reference stations away from the zone of local tectonic activity. Even so, few valid examples of geomagnetic change related to stress have been reported, although the expected magnitude of the effect is as high as a few gamma.

Perhaps the best known success is the correlation between local magnetic changes and creep events on the San Andreas fault observed by Breiner (thesis, Stanford University ; 1967), even though, at the same time, he failed to detect any relationship between geomagnetic variations and earthquakes in the same region. More recently, Davis and Stacey (Nature, 240, 348 ; 1972) have further demonstrated that "the piezomagnetic effect is observable on a tectonic scale" by observing geomagnetic changes associated with the increasing water load in the Talbingo Dam of south-east Australia. Convincing observations of local magnetic changes preceding and accompanying eruptions of the Ruapehu and Ngauruhoe volcanoes of New Zealand have also been reported by Johnston and Stacey (J. Geophys, Res., 74, 6541; 1969 , and Nature, 224, 1289 ; 1969). On the other hand, Yokoyama (The Surveillance and Prediction of Volcanic Activity, UNESCO ; 1971) has attributed geomagnetic changes associated with Japanese volcanoes to local changes of temperature rather than to stress.

In an attempt to find further examples of the volcanomagnetic effect, Davis et al. (Science, 180, 73; 1973) have investigated Kilauea Volcano in Hawaii. On the face of it, Kilauea offers a good opportunity for this type of work because it frequently erupts, highly magnetic basalts surround it, and the existence of the Hawaiian Volcano Observatory close by gives a good "logistic basis" for an experiment involving repeated observations of an instrumental array. Davis and his colleagues thus constructed a linked array of three total field magnetometers comprising one local station close to Kilauea and two more distant reference stations (10 km and $43 \mathrm{~km}$ away, respectively).

Recording of the geomagnetic field was continued for a (broken) period of 12 months in conjunction with the existing programme of tilt and strain measurement in Kilauea which has already demonstrated inflation of the summit before eruptions and deflation during flank eruptions. The geo- magnetic changes to be expected from such tilts can easily be estimated. Thus a tilt of about $10^{-4} \mathrm{rad}$, implying a strain of about $10^{-4}$, would give a stress of about 30 bar in basalt, assuming perfect elasticity. Titanomagnetite of the composition indicated by the relevant Curie points would then give an expected change in magnetization of 2 parts in $10^{4}$ per bar of stress, which corresponds to an absolute change of at least $3 \times 10^{-5}$ e.m.u. and a resulting magnetic anomaly of the order of 10 gamma if the basalt is magnetic to a substantial depth.

But although such magnetic changes were observed in one of the experiments, they failed to correlate directly with the tilt records, and in a second experiment, no magnetic anomalies developed at all. Thus when the local station was at Uwekahuma, close to the Hawaiian Volcano Observatory, brief excursions of the magnetic field occurred. These have a very slight correlation with small irregularities in the tilt records, but the effect is very localized. When the local station was moved to a site closer to the source of deformation, no magnetic anomalies developed at all even when tilting was violent.

The search for the volcanomagnetic effect from Kilauea was thus unrewarding, although even this negative result leads to a definite conclusion. It is clear that during a prolonged deformation of Kilauea the volcano cannot support any large scale pattern of shear stress but only transient, localized stresses accompanying local readjustments. Davis et al. do not discuss the effects of temperature at all ; but it is interesting to note that their conclusion based on experiment was also reached by Yokoyama who argued the general case based on a consideration of temperature.

\title{
Scattering in the Lower Mantle
}

IRREGULAR structures acting as seismic wave scatterers in the Earth are generally difficult, if not impossible, to detect because the small amount of scattered energy is easily obscured by the primary wave. But in seismic shadow zones the conditions are more favourable. For example, in the PKP shadow zone caused by the presence of the Earth's core, the first phase to be expected on the basis of the most simple core model is PKIKP, the phase refracted through the inner core. In practice, however, precursors to PKIKP are observed in the approximate epicentral distance range $125^{\circ}$ to $143^{\circ}$ - a phenomenon for which an explanation is required.

In fact, there are several possible (though not mutually exclusive) explanations, including more complicated core models energy diffracted from the PKP caustic surface in a limited range near the caustic, scattering from the Bullen $D^{\prime \prime}$ layer and in other parts of the mantle near the caustic surface, and scattering in the lower mantle beneath the source region. In next Monday's Nature Physical Science (May 28), Doornbos and Vlaar present an analysis of precursor data from the Norwegian seismic array (NORSAR) which provides evidence for all of these explanations except that involving a more complex core structure. In addition, they have been able to determine the locations of some of the inferred scattering zones.

From a spectral analysis of events in the Solomon and Fiji Islands regions during 1971 and 1972, Doornbos and Vlaar have been able to divide the precursors into three distinct classes based on differences in slowness $(\mathrm{d} T / \mathrm{d} \Delta)$ and the azimuth of the direction of wave propagation. The first set comprises first arrivals in the range $143^{\circ}-138^{\circ}$ which have an average $\mathrm{d} T / \mathrm{d} \Delta$ value of about $3.3 \mathrm{~s}$ per degree and deviation from the true azimuth of less than $2^{\circ}$; the second set includes phases with anomalously low $\mathrm{d} T / \mathrm{d} \Delta$ values in the range 1.6-3.0 $\mathrm{s}$ per degree and large azimuthal deviations in the range $10^{\circ}$ to $40^{\circ}$; and the third set consists of phases with anomalously high $\mathrm{d} T / \mathrm{d} \Delta$ values between 3.4 and $4.0 \mathrm{~s}$ per degree but azimuthal deviations within $5^{\circ}$.

There seems no reason to doubt that the precursors in the first set, at least those between $143^{\circ}$ and $140^{\circ}$, are due to diffraction from the caustic as predicted by theory. Neither diffraction nor core complexity is, however, adequate to explain the second and third sets. If the phases in the second set are taken to be generated by scattering near the caustic surface, their characteristics may be used to determine the positions of the proposed scattering regions. Thus Doornbos and Vlaar conclude that scattering structures exist in the lower mantle at distances of $250 \mathrm{~km}, 500 \mathrm{~km}$ and $600 \mathrm{~km}$ from the core-mantle boundary. The errors on these locations are difficult to estimate, but are unlikely to exceed $100 \mathrm{~km}$ for the first one and $150 \mathrm{~km}$ for the other two. The uncertainties are thus not small but neither are they large enough to explain the depth spread of the locations.

The large $d T / d \Delta$ values for the precursors in the third set rule out an origin on the receiver side of the mantle but may be attributed to scattering structures on the source side. Accordingly, Doornbos and Vlaar also locate structures at distances of $200 \mathrm{~km}, 800$ $\mathrm{km}$ and $900 \mathrm{~km}$ from the core-mantle boundary although in these cases the estimated errors amount to several hundred kilometres. 